\title{
Almost Tight Lower Bounds for Hard Cutting Problems in Embedded Graphs
}

\author{
Vincent Cohen-Addad \\ Sorbonne Universités, UPMC Univ Paris 06, CNRS, LIP6, Paris, France \\ vcohenad@gmail.com
}

\section{Éric Colin de Verdière}

Université Paris-Est, LIGM, CNRS, ENPC, ESIEE Paris, UPEM, Marne-la-Vallée, France

eric.colindeverdiere@u-pem.fr

\author{
Dániel Marx \\ Institute for Computer Science and Control, Hungarian Academy of Sciences (MTA SZTAKI), \\ Budapest, Hungary \\ dmarx@cs.bme.hu
}

\begin{abstract}
Arnaud de Mesmay
Univ. Grenoble Alpes, CNRS, Grenoble INP ${ }^{1}$, GIPSA-lab, 38000 Grenoble, France arnaud.de-mesmay@gipsa-lab.fr
\end{abstract}

\begin{abstract}
We prove essentially tight lower bounds, conditionally to the Exponential Time Hypothesis, for two fundamental but seemingly very different cutting problems on surface-embedded graphs: the Shortest Cut Graph problem and the Multiway Cut problem.

A cut graph of a graph $G$ embedded on a surface $S$ is a subgraph of $G$ whose removal from $S$ leaves a disk. We consider the problem of deciding whether an unweighted graph embedded on a surface of genus $g$ has a cut graph of length at most a given value. We prove a time lower bound for this problem of $n^{\Omega(g / \log g)}$ conditionally to ETH. In other words, the first $n^{O(g)}$-time algorithm by Erickson and Har-Peled [SoCG 2002, Discr. Comput. Geom. 2004] is essentially optimal. We also prove that the problem is $\mathrm{W}[1]$-hard when parameterized by the genus, answering a 17-year old question of these authors.

A multiway cut of an undirected graph $G$ with $t$ distinguished vertices, called terminals, is a set of edges whose removal disconnects all pairs of terminals. We consider the problem of deciding whether an unweighted graph $G$ has a multiway cut of weight at most a given value. We prove a time lower bound for this problem of $n^{\Omega\left(\sqrt{g t+g^{2}} / \log (g t)\right)}$, conditionally to ETH, for any choice of the genus $g \geq 0$ of the graph and the number of terminals $t \geq 4$. In other words, the algorithm by the second author [Algorithmica 2017] (for the more general multicut problem) is essentially optimal; this extends the lower bound by the third author [ICALP 2012] (for the planar case).

Reductions to planar problems usually involve a grid-like structure. The main novel idea for our results is to understand what structures instead of grids are needed if we want to exploit optimally a certain value $g$ of the genus.
\end{abstract}

2012 ACM Subject Classification Mathematics of computing $\rightarrow$ Graphs and surfaces; Mathematics of computing $\rightarrow$ Graph algorithms

Keywords and phrases Cut graph, Multiway cut, Surface, Lower bound, Parameterized Complexity, Exponential Time Hypothesis

Digital Object Identifier 10.4230/LIPIcs.SoCG.2019.27

Funding Vincent Cohen-Addad: Ce projet a bénéficié d'une aide de l'État gérée par l'Agence Nationale de la Recherche au titre du Programme FOCAL portant la référence suivante : ANR-18CE40-0004-01.

1 Institute of Engineering Univ. Grenoble Alpes

(c) (i) $\odot$ Vincent Cohen-Addad, Éric Colin de Verdière, Dániel Marx, and Arnaud de Mesmay; 35th International Symposium on Computational Geometry (SoCG 2019). Editors: Gill Barequet and Yusu Wang; Article No. 27; pp. 27:1-27:16

Leibniz International Proceedings in Informatics LIPICS Schloss Dagstuhl - Leibniz-Zentrum für Informatik, Dagstuhl Publishing, Germany 
Éric Colin de Verdière: Partially supported by the French ANR project ANR-17-CE40-0033 (SoS). Dániel Marx: Supported by ERC Consolidator Grant SYSTEMATICGRAPH (No. 725978).

Arnaud de Mesmay: Partially supported by the French ANR projects ANR-16-CE40-0009-01 (GATO), ANR-18-CE40-0004-01 (FOCAL) and the CNRS PEPS project COMP3D.

Acknowledgements We are grateful to the anonymous referees for a careful reading of the paper and many helpful suggestions.

\section{Introduction}

During the past decade, there has been a flurry of works investigating the complexity of solving exactly optimization problems on planar graphs, leading to what was coined as the "square root phenomenon" by the third author [27]: many problems turn out to be easier on planar graphs, and the improvement compared to the general case is captured exactly by a square root. For instance, problems solvable in time $2^{O(n)}$ in general graphs can be solved in time $2^{O(\sqrt{n})}$ in planar graphs, and similarly, in a parameterized setting, FPT problems admitting $2^{O(k)} n^{O(1)}$-time algorithms or $\mathrm{W}[1]$-hard problems admitting $n^{O(k)}$-time algorithms can often be sped up to $2^{\tilde{O}(\sqrt{k})} n^{O(1)}$ and $n^{\tilde{O}(\sqrt{k})}$, respectively, when restricted to planar graphs. We have many examples where matching upper bounds (algorithms) and lower bounds (complexity reductions) show that indeed the best possible running time for the problems has this form. On the side of upper bounds, the improvement often stems from the fact that planar graphs have (recursive) planar separators of size $O(\sqrt{n})$, and the theory of bidimensionality provides an elegant framework for a similar speedup in the parameterized setting for some problems [12]. However, in many cases these algorithms rely on highly problem-specific arguments $[6,28,21,30,2,23,14]$. The lower bounds are conditional to the Exponential Time Hypothesis (ETH) of Impagliazzo, Paturi, and Zane [19] and follow from careful reductions from problems displaying this phenomenon, e.g., PLANAR 3-Coloring, $k$-Clique, or Grid Tiling. We refer to the recent book [9] for precise results along these lines.

While the theme of generalizing algorithms from planar graphs to surface-embedded graphs has attracted a lot of attention, and has flourished into an established field mixing algorithmic and topological techniques (see [7]), the same cannot be said at all of the lower bounds. Actually, up to our knowledge, there are very few works explicitly establishing algorithmic lower bounds based on the genus of the surfaces on which a graph is embedded, or even just hardness results when parameterized by the genus - the only ones we are aware of are the exhaustive treatise [29] of the third author and Pilipczuk on SUBGRAPH ISOMORPHISM, where some of the hardness results feature the genus of the graph, the lower bounds of Curticapean and the third author [8] on the problem of counting perfect matchings and the work of Chen et al. [1].

In this work, we address this surprising gap by providing lower bounds conditioned on ETH for two fundamental yet seemingly very different cutting problems on surface-embedded graphs: the Shortest Cut Graph problem and the Multiway Cut problem. In both cases, our lower bounds match the best known algorithms up to a logarithmic factor in the exponent. We believe that the tools that we develop in this paper could pave the way towards establishing lower bounds for other problems on surface-embedded graphs.

The shortest cut graph problem. A cut graph of an edge-weighted graph $G$ cellularly embedded on a surface $S$ is a subgraph of $G$ that has a unique face, which is a disk. Computing a shortest cut graph is a fundamental problem in algorithm design, as it is often 
easier to work with a planar graph than with a graph embedded on a surface of positive genus, since the large toolbox that has been designed for planar graphs becomes available. Furthermore, making a graph planar is useful for various purposes in computer graphics and mesh processing, see, e.g., [34]. Be it for a practical or a theoretical goal, a natural measure of the distortion induced by the cutting step is the length of the topological decomposition.

Thus, the last decade has witnessed a lot of effort on how to obtain efficient algorithms for the problems of computing short topological decompositions, see for example the survey [7]. For the shortest cut graph problem, Erickson and Har-Peled [13] showed that the problem is NP-hard when the genus is considered part of the input and gave an exact algorithm running in time $n^{O(g)}$, where $n$ is the size of the input graph and $g$ the genus of the surface, together with an $O\left(\log ^{2} g\right)$-approximation running in time $O\left(g^{2} n \log n\right)$. The first and fourth authors [5] gave a $(1+\varepsilon)$-approximation algorithm running in time $O\left(f(\varepsilon, g) n^{3}\right)$, where $f$ is some explicit computable function. Whether it is possible to improve upon the exact algorithm of Erickson and Har-Peled by designing an FPT algorithm for the problem, namely an exact algorithm running in time $f(g) n^{O(1)}$, has been raised by these authors $[13$, Conclusion] and has remained an open question over the last 17 years.

In this paper, we solve this question by proving that the result of Erickson and Har-Peled cannot be significantly improved. We indeed show a lower bound of $n^{\Omega(g / \log g)}$ (for the associated decision problem, even in the unweighted case) assuming the Exponential Time Hypothesis (ETH) of Impagliazzo et al. [19] (see Definition 3), and also prove that the problem is $\mathrm{W}[1]$-hard:

Theorem 1. Let us consider the SHORTEST CUT GRAPH problem: Given an unweighted graph $G$ with $n$ vertices cellularly embedded on an orientable surface of genus $g$, and an integer $\lambda$, decide whether $G$ admits a cut graph of length at most $\lambda$.

1. This problem is W[1]-hard when parameterized by $g$.

2. Assuming ETH, there exists a universal constant $\alpha_{\mathrm{CG}}$ such that for any fixed integer $g \geq 2$, there is no algorithm solving all the SHORTEST CUT GRAPH instances of genus at most $g$ in time $O\left(n^{\alpha_{\mathrm{CG}} \cdot g / \log g}\right)$.

(In the second item, the constraint $g \geq 2$ is just here to ensure that $g / \log g$ is well-defined.)

The multiway cut problem. The second result of our paper concerns the Multiway CUT problem (also known as the Multiterminal Cut problem). Given an edge-weighted graph $G$ together with a subset $T$ of $t$ vertices called terminals, a multiway cut is a set of edges whose removal disconnects all pairs of terminals. Computing a minimum-weight multicut is a classic problem that generalizes the minimum $s-t$ cut problem and some closely related variants have been actively studied since as early as 1969 [18]. On general graphs, while the problem is polynomial-time solvable for $t=2$, it becomes NP-hard for any fixed $t \geq 3$, see [10]. In the case of planar graphs, it remains NP-hard if $t$ is arbitrarily large, but can be solved in time $2^{O(t)} n^{O(\sqrt{t})}$, where $n$ is the number of vertices and edges of the graph [21], and a lower bound of $n^{\Omega(\sqrt{t})}$ was proved (conditionally on ETH) by the third author [26]. A generalization to higher-genus graphs was recently obtained by the second author [6] who devised an algorithm running in time $\left.f(g, t) \cdot n^{O\left(\sqrt{g t+g^{2}}\right.}\right)$ in graphs of genus $g$, for some function $f$ (actually, for the more general MulticuT problem). If one allows some approximation, this can be significantly improved: three of the authors recently provided a $(1+\varepsilon)$-approximation algorithm running in time $f(\varepsilon, g, t) \cdot n \log n[3]$.

We prove a lower bound of $n^{\Omega\left(\sqrt{g t+g^{2}} / \log (g t)\right)}$ for the associated decision problem, even in the unweighted case, which almost matches the aforementioned best known upper bound, and generalizes the lower bound of the third author [26] for the planar case. Actually, we 
prove a lower bound that holds for any value of the integers $g$ and $t$ as long as $t \geq 4$. The precise theorem is the following, where we use $g^{*}$ to denote $\max (2, g)$ so that the quantities are well-defined for $g=0$ and $g=1$ :

- Theorem 2. Let us consider the MULTIWAY CUT problem: Given an unweighted graph $G$, a set $T$ of vertices, and an integer $\lambda$, decide whether there exists a multiway cut of $(G, T)$ of value at most $\lambda$.

Assuming ETH, there exists a universal constant $\alpha_{\mathrm{MC}}$ such that for any fixed choice of integers $g \geq 0$ and $t \geq 4$, there is no algorithm that decides all the MultiwaY Cut instances $(G, T, \lambda)$ for which $G$ is embeddable on the orientable surface of genus $g$ and $|T| \leq t$, in time $O\left(n^{\alpha_{\mathrm{MC}}} \sqrt{g^{*} t+g^{* 2}} / \log \left(g^{*} t\right)\right)$.

Note that taking $g=0$ in this theorem yields lower bounds for the Planar MultiwaY Cut problem, and recovers, up to a logarithmic factor, the lower bounds obtained by the third author [26] for that problem. In the opposite regime, we also prove W[1]-hardness with respect to the genus for instances with 4 terminals, see Proposition 11. We remark that $t=2$ corresponds to the minimum cut problem, which is polynomial-time solvable, so a lower bound on $t$ is necessary. While the last remaining case, for $t=3$, is known to be NP-hard [10], our techniques do not seem to encompass it, and we leave its parameterized complexity with respect to the genus as an open problem.

- Remark. Parameterized lower bounds in the literature often have the form "assuming ETH, there is no $f(k) n^{o(h(k))}$ algorithm to solve problem $\mathrm{X}$, for any function $f$ ", where $h$ is some specific dependency on the parameter. The lower bounds that we prove in Theorems 1 and 2 are instead of the form "assuming ETH, there exists a universal constant $\alpha$ such that for any fixed $k$, there is no $O\left(n^{\alpha h(k)}\right)$ algorithm to solve problem X". The latter lower bounds imply the former: indeed, $f(k) n^{o(h(k))}=O\left(n^{\alpha h(k)}\right)$ for a fixed $k$. Our results are stronger, concerning instances for any fixed $k$. Moreover, lower bounds with two parameters are difficult to state with $o()$ notation. The statement of Theorem 2 handles every combination of the two parameters in a completely formal way.

Main ideas of the proof. What is a good starting problem to prove hardness results for surface-embedded graphs? For planar graphs, the GRID TILING problem of the third author [24] has now emerged as a convenient, almost universal, tool to establish parameterized hardness results and precise lower bounds based on ETH. A similar approach, based on constraint satisfaction problems (CSPs) on $d$-dimensional grids, was used by the third author and Sidiropoulos [31] to obtain lower bounds for geometric problems on low-dimensional Euclidean inputs (see also [11] for a similar framework for geometric intersection graphs). However, these techniques do not apply directly for the problems that we consider. Indeed, the bounds implied by these approaches are governed by the treewidths of the underlying graphs and are of the type $n^{\Omega(\sqrt{p})}$ or $n^{\Omega\left(p^{1-1 / d}\right)}$ respectively, where $p$ is the parameter of interest and $d$ the dimension of the grid in the latter case. In contrast, here, we are looking for bounds of the form $n^{\Omega^{*}(p)}$ (while this is not apparent from looking at Theorem 2, this also turns out to be the main regime of interest for the Multiway Cut problem).

Our first contribution, in Section 3, is to introduce a new hard problem for embedded graphs, which is versatile enough to be used as a starting point to obtain lower bounds for both the Shortest Cut Graph and the Multiway Cut problem (and hopefully others). It is a variant of the GRID Tiling problem which we call 4-Regular Graph Tiling; in a precise sense, it generalizes the GRID TILING problem to allow for embedded 4-regular graphs different from the planar grid to be used as the structure graph of the problem. We 
show that a CSP instance with $k$ binary constraints can be simulated by a 4-REGULAR Graph Tiling instance with parameter $k$. A result of the third author [25] shows that, assuming the ETH, such CSP instances cannot be solved in time $f(k) n^{\Omega(k / \log k)}$, giving a similar lower bound for 4-REgular Graph Tiling (Theorem 9).

We then establish in Sections 4 and 5 the lower bounds for the Shortest Cut GrapH and "one half" of the lower bound for MultiwAY CUT, namely, for the regime where the genus dominates the number of terminals. Both reductions proceed from 4-REGULAR GRAPH TILING and use as a building block an intricate set of cross gadgets originally designed by the third author [26] for his hardness proof of the Planar Multiway Cut problem. While it does not come as a surprise that these gadgets are useful for more general non-planar Multiway Cut instances, it turns out that via basic planar duality, they also provide exactly the needed technical tool for establishing the hardness of SHORTEST Cut GrapH.

In order to establish the "second half" of the lower bound in Theorem 2, in the regime where the number of terminals dominates the genus, we use a similar strategy in Section 6 but bypass the use of the 4-REgular GRAPH TILING problem. Instead, we rely directly on the aforementioned theorem of the third author on the parameterized hardness of CSPs, which we apply not to a family of expanders, but to blow-ups of expanders, i.e., expanders where vertices are replaced by grids of a well-chosen size. This size is prescribed exactly by the tradeoff between the genus and the number of terminals, as described with the two integers $g$ and $t$ in Theorem 2. The key property of these blow-ups is that their treewidth is $\mathrm{tw}=\Theta(\sqrt{g t})$ and thus the $n^{\Omega(\mathrm{tw} / \log \mathrm{tw})}$ lower bound on the complexity of CSPs with these blow-ups as primal graphs yields exactly the target lower bound. The reduction from CSPs to Multiway Cut is carried out in Proposition 14 and also relies on cross gadgets.

There just remains to combine Propositions 11 and 14 to obtain Theorem 2. This is easy and we refer to the full version [4, Section 7] for the proof.

Note that while Theorem 2 does not use an embedded graph as an input, we can find an embedding of a graph on a surface with minimum possible genus in $f(g) n$ time [20, 32]. Thus, the same hardness result holds in the embedded case and the question is not about whether we are given the embedding or not.

\section{Preliminaries}

Graphs on surfaces. For standard definitions for graphs embedded on surfaces, we refer to the classic textbook of Mohar and Thomassen [33] and the fullversion [4, Section 2]. In this article, all surfaces are compact, connected, and orientable.

The Exponential Time Hypothesis. Our lower bounds are conditioned on the Exponential Time Hypothesis (ETH), which was conjectured in [19].

- Conjecture 3 (Exponential Time Hypothesis [19]). There exists a positive real value $s>0$ such that 3-CNF-SAT, parameterized by $n$, has no $2^{\text {sn }}(n+m)^{O(1)}$-time algorithm (where $n$ denotes the number of variables and $m$ denotes the number of clauses).

We refer to the survey [22] for background and discussion of this conjecture.

Expanders and their treewidth. We will rely on the following classical lemmas about expander graphs and their treewidth. A family $\mathcal{G}$ of graphs is dense if for any $n>0$, there exists a graph in $\mathcal{G}$ with $\Theta(n)$ vertices (where the $\Theta()$ hides a universal constant). The other definitions and the proofs are included in the full version [4, Section 2] 
- Lemma 4. There exists a dense family $\mathcal{H}$ of bipartite four-regular expanders.

- Lemma 5. Every d-regular graph $G$ satisfies $\operatorname{tw}(G) \geq\lfloor|V(G)| \cdot(1-\lambda(G) / d) / 8\rfloor$.

Constraint satisfaction problems. A binary constraint satisfaction problem is a triple $(V, D, C)$ where

- $V$ is a set of variables,

- $D$ is a domain of values,

- $R$ is a set of constraints, $\left\{c_{1}, \ldots, c_{q}\right\}$, which are all pairs $\left\langle s_{i}, R_{i}\right\rangle$, where $s_{i}$ is a pair of variables called the scope, and $R_{i}$ is a subset of $D^{2}$ called the relation.

All the constraint satisfaction problems (CSPs) in this paper will be binary, and thus we will omit the adjective binary.

A solution to a constraint satisfaction problem instance is a function $f: V \rightarrow D$ such that for each constraint $c_{i}$ with $s_{i}=\left(v_{1}, v_{2}\right)$, the pair $f\left(v_{1}, v_{2}\right)$ is a member of $R_{i}$. An algorithm decides a CSP instance $I$ if it outputs true if and only if that instance admits a solution.

The primal graph of a CSP instance $I=(V, D, C)$ is a graph with vertex set $V$ such that distinct vertices $u, v \in V$ are adjacent if and only if there is a constraint whose scope contains both $u$ and $v$.

The starting points for the reductions in this paper are the following two theorems, which state in a precise sense that the treewidth of the primal graph of a binary CSP establishes a lower bound on the best algorithm to decide it.

- Theorem 6 ([17, 16]). Let $\mathcal{G}$ be an arbitrary class of graphs with unbounded treewidth. Let us consider the problem of deciding the binary CSP instances whose primal graph, $G$, lies in $\mathcal{G}$. This problem is W[1]-hard parameterized by the treewidth.

- Theorem 7 ([25]). Assuming ETH, there exists a universal constant $\alpha_{\mathrm{CSP}}$ such that for any fixed primal graph $G$ with $\operatorname{tw}(G) \geq 2$, there is no algorithm deciding the binary CSP instances whose primal graph is $G$ in time $O\left(|D|^{\alpha_{\mathrm{CSP}} \cdot \operatorname{tw}(G) / \log \operatorname{tw}(G)}\right)$.

The first theorem is due to Grohe et al. [17] (see also Grohe [16]). The second one follows from the work of the third author [25]. Since this statement differs from the main theorem of [25], we explain in the full version [4, Section 2] how to obtain it.

Cross gadgets. We rely extensively on the following intricate family of gadgets introduced by the third author in his proof of hardness of PlanAR Multiway Cut [26], which we call cross gadgets. Let $\Delta$ be an integer. The gadgets always have the form of a planar graph $G_{S}$ embedded on a disk, with $4 \Delta+8$ distinguished vertices on its boundary, which are, in clockwise order, denoted by

$$
U L, u_{1}, \ldots, u_{\Delta+1}, U R, r_{1}, \ldots, r_{\Delta+1}, D R, d_{\Delta+1}, \ldots d_{1}, D L, \ell_{\Delta+1}, \ldots, \ell_{1} .
$$

The embedding is chosen so that the boundary of the disk intersects the graph precisely in this set of distinguished vertices; the interior of the edges lie in the interior of the disk. We consider the vertices $U L, U R, D R$, and $D L$ as terminals in that gadget, and thus a multiway cut $M$ of the gadget is a subset of the edges of $G_{S}$ such that $G_{S} \backslash M$ has at least four components, and each of the terminals is in a distinct component. We say that a multiway cut $M$ of the gadget represents the pair $(i, j) \in[\Delta]^{2}$ (where, as usual, $[\Delta]$ denotes the set $\{1, \ldots, \Delta\})$ if $G_{S} \backslash M$ has exactly four components that partition the distinguished vertices into the following classes:

$$
\begin{array}{rr}
\left\{U L, u_{1}, \ldots, u_{j}, \ell_{1}, \ldots, \ell_{i}\right\} & \left\{U R, u_{j+1}, \ldots \Delta+1, r_{1}, \ldots, r_{i}\right\} \\
\left\{D L, d_{1}, \ldots d_{j}, \ell_{i+1}, \ldots, \ell_{\Delta+1}\right\} & \left\{D R, d_{j+1}, \ldots, d_{\Delta+1}, r_{i+1}, \ldots r_{\Delta+1}\right\}
\end{array}
$$



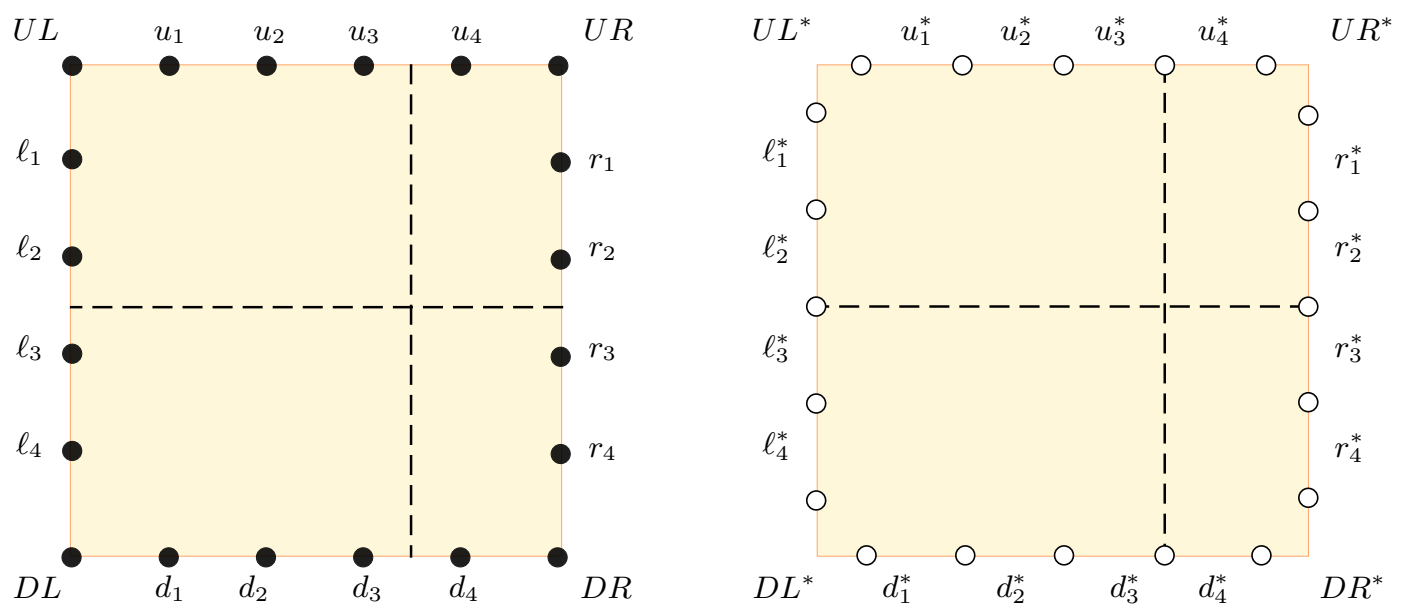

Figure 1 Left: a cross gadget $G_{S}$ for $\Delta=3$. The dashed line indicates a multiway cut that represents the pair $(2,3)$. Right: a dual cross gadget $G_{S}^{*}$ for $\Delta=3$. The dashed lined is a dual multiway cut that represents the pair $(2,3)$.

We remark that, as in the original article [26], the notation $(i, j)$ is in matrix form. We will use the same convention throughout this paper, especially in Section 3.

The boundary of a cross gadget and a multiway cut representing a pair are pictured on Figure 1, left. The properties that we require are summarized in the following lemma:

- Lemma 8 ([26, Lemma 2]). Given a subset $S \subseteq[\Delta]^{2}$, we can construct in polynomial time a planar gadget $G_{S}$ with poly $(\Delta)$ unweighted edges and vertices, and an integer $D_{1}$ such that the following properties hold:

i. For every $(i, j) \in S$, the gadget $G_{S}$ has a multiway cut of weight $D_{1}$ representing $(i, j)$.

ii. Every multiway cut of $G_{S}$ has weight at least $D_{1}$.

iii. If a multiway cut of $G_{S}$ has weight $D_{1}$, then it represents some $(i, j) \in S$.

Note that in [26], the third author uses weights to define the gadgets, but as he explains at the end of the introduction, the weights are polynomially large integers and thus can be emulated with parallel unweighted edges.

In the following, we will also use the dual of the graph $G_{S}$ as one of our gadgets, yielding a dual cross gadget $G_{S}^{*}$ (see Figure 1). Its properties mirror exactly the ones of cross gadgets in a dual setting, we refer to the full version [4, Section 2] for details.

\section{The 4-regular graph tiling problem}

We introduce the problem 4-REgular GraPh TILING which will be used as a basis to prove the reductions involved in Theorems 1 and 2 .

4-Regular Graph TiLing

Input: Integers $k, n$; a four-regular graph $\Gamma$ on $k$ vertices where the edges are labeled by $U, D, L, R$ in a way that each vertex is incident to exactly one of each label; for each vertex $v$, a non-empty set $S_{v} \subseteq[n] \times[n]$.

Output: For each vertex $v$, a value $s_{v} \in S_{v}$ such that if $s_{v}=(i, j)$,

1. the first coordinate of $s_{L(v)}$ and $s_{R(v)}$ is $i$, and

2. the second coordinate of $s_{U(v)}$ and $s_{D(v)}$ is $j$, 


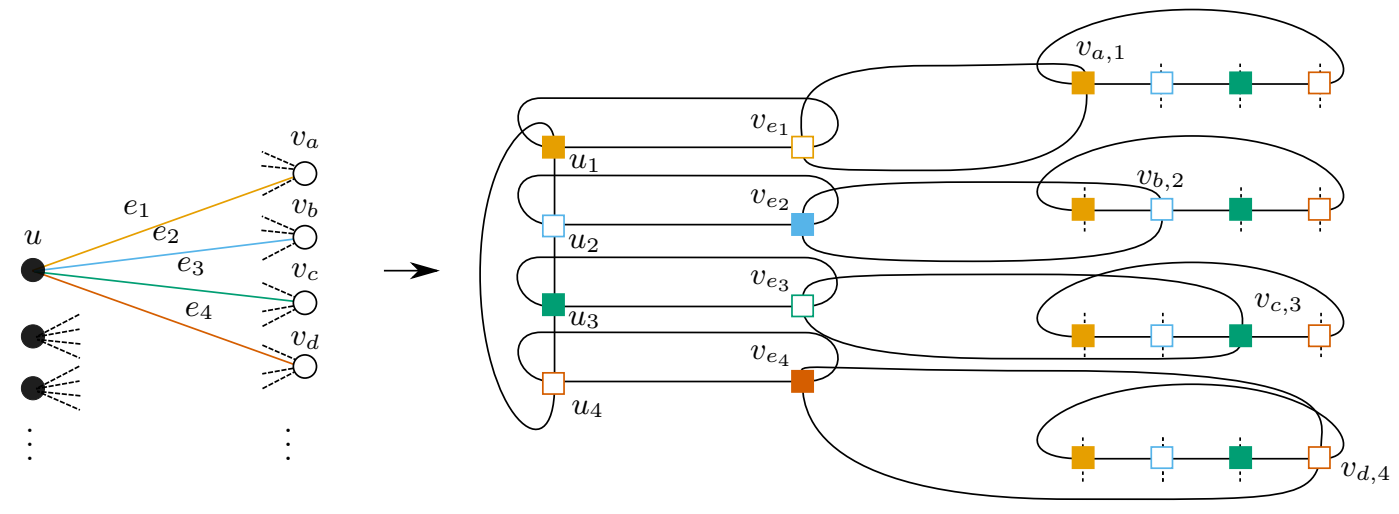

Figure 2 The reduction in the proof of Theorem 9. The bipartition on both sides are represented by hollow/solid vertices. The colors represent the 4-coloring of the edges, and the labels of the edges are suggested by their orientation, i.e., edges entering vertices vertically are labeled $U$ or $D$, while edges entering vertices horizontally are labeled $L$ or $R$.

where $U(v), D(v), L(v)$, and $R(v)$ denote the vertex of the graph $\Gamma$ connected to $v$ via an edge labeled respectively by $U, D, L$, and $R$.

We call the two conditions above the compatibility conditions of the 4-REGULAR GrAPH TILING instance. The graph in the input is allowed to have parallel edges. It is easy to see that the Grid Tiling problem [24] is a special case of 4-REgular Graph Tiling.

In this section, we prove a larger lower bound for this more general problem: we prove an $n^{\Omega(k / \log k)}$ lower bound, conditionally to ETH, for 4-REgular GRAPH TILING, even when the problem is restricted to bipartite instances and when fixing $k$. We also show that it is $\mathrm{W}[1]$-hard when parameterized by the integer $k$ (even for bipartite instances). Precisely:

\section{- Theorem 9.}

1. The 4-Regular Graph Tiling problem restricted to instances whose underlying graph is bipartite, parameterized by the integer $k$, is W[1]-hard.

2. Assuming ETH, there exists a universal constant $\alpha_{\mathrm{GT}}$ such that for any fixed integer $k \geq 2$, there is no algorithm that decides all the 4-REGULAR GRAPH TILING instances whose underlying graph is bipartite and has at most $k$ vertices, in time $O\left(n^{\alpha_{\mathrm{GT}} \cdot k / \log k}\right)$.

The analogous result for GRID TILING by the third author [24] embeds the $k$-CLIQUE problem in a $k \times k$ grid. Here we start from a hardness result for 4-regular binary CSPs that follows from Theorem 7 and directly represent the problem as a 4-REgular GRAPH Tiling instance by locally replacing each variable and each binary constraint in an appropriate way.

Proof. In the proof, we will use the well-known fact that a $d$-regular bipartite graph $G$ can be properly edge-colored with $d$ colors. This is proved by induction on $d$ : The case $d=0$ is trivial; in general, take a perfect matching of $G$, which exists by Hall's marriage theorem; color the edges with color $d$; the subgraph of $G$ made of the uncolored edges satisfies the induction hypothesis with $d-1$, so it admits a proper edge-coloring with $d-1$ colors; thus $G$ has a proper edge-coloring with $d$ colors. This also implies that computing such a proper edge-coloring takes polynomial time.

The proof of the theorem proceeds by a reduction from the binary CSP instances involved in Theorems 6 and 7. Starting from a binary CSP instance $I=(V, D, C)$ whose primal graph is $P$, a 4-regular bipartite graph, we define an instance of 4-REgUlar Graph Tiling, $\left(k, n,\left\{S_{i}\right\}, \Gamma\right)$, in the following way. 
1. We set $n=|D|$ and $k=6|V|$.

2. We find a proper edge coloring of $P$ with 4 colors, as indicated above.

3. Denoting by $V_{1}$ and $V_{2}$ the two subsets of vertices of $P$ corresponding to the bipartition of $P$, for each vertex $u$ of $V_{1}$, we create four vertices $u_{1}, u_{2}, u_{3}, u_{4}$ in $\Gamma$ which we connect in a cycle in this order using two $U$ and two $D$ edges. Similarly, for each vertex $v$ of $V_{2}$, we create four vertices $v_{1}, v_{2}, v_{3}, v_{4}$ in $\Gamma$ which we connect in a cycle in this order using two $R$ and two $L$ edges.

4. For each edge $e=u v$ labeled with a color $i$, where $u \in V_{1}$ and $v \in V_{2}$, we create one vertex $v_{e}$ in $\Gamma$, which is connected to $u_{i}$ via two edges, one labeled $R$ and one labeled $L$, and to $v_{i}$ via two edges, one labeled $U$ and one labeled $D$.

5. For each vertex $u_{i}$ or $v_{i}$ of $\Gamma$ coming from a vertex of $P$, the corresponding subset $S_{u_{i}}$ or $S_{v_{i}}$ is set to be $\operatorname{Diag}([n]):=\{(x, x) \mid x \in[n]\}$.

6. For each vertex $v_{e}$ of $\Gamma$ coming from an edge $e=u v$ of $P$, where $u \in V_{1}$ and $v \in V_{2}$, the corresponding subset $S_{e}$ is set to be the relation corresponding to $e$.

See Figure 2 for an illustration of this reduction. We claim that the graph $\Gamma$ is bipartite: The bipartition is obtained by picking for one side the odd-numbered $u$ and $v$ vertices and the $v_{e}$ vertices for $e$ labeled by an even color, and for the other side the even-numbered $u$ and $v$ vertices and the $v_{e}$ vertices for $e$ labeled by an odd color. It follows from the construction that this is a bipartition.

We claim that this instance of 4-REgUlar GRAPH TILING is satisfiable if and only if $I$ is satisfiable. Indeed, if $I$ is satisfiable, the truth assignment $f$ for $I$ can be used to find the values for the $s_{i}$ in the following way. For a vertex $u_{i}$ or $v_{i}$ of $\Gamma$ coming from a vertex $v$ of $P$, the value $s_{u_{i}}$ or $s_{v_{i}}$ can be chosen to be $(f(v), f(v))$. For a vertex $v_{e}$ of $\Gamma$ coming from an edge $e=u v$ of $P$ where $u \in V_{1}$ and $v \in V_{2}$, the value of $s_{e}$ can be chosen to be $(f(u), f(v))$. The compatibility conditions are trivially fulfilled. In the other direction, the values $s_{v_{i}}$ for the four vertices of $\Gamma$ coming from a vertex $v$ of $P$ are identical and of the form $(x, x)$. Choosing $x$ as the truth assignment for $v$ in $I$ yields a solution to the CSP $I$.

We thus have a linear-time reduction from binary CSP, restricted to instances $I$ whose primal graph has $|V|$ vertices, is four-regular and is bipartite, to instances of 4-REGULAR Graph Tiling on a bipartite graph with $6|V|$ vertices. Combined with Theorem 6 applied to the infinite family $\mathcal{H}$ of four-regular bipartite expanders output by Lemma 4 and Lemma 5 relating their treewidth to their number of vertices, this proves the first item of the theorem. For the second item, we fix an integer $k$; by Lemma 4, there exists a constant $c$ so that if $k \geq c$, there exists a four-regular bipartite expander $G$ with expansion constant $1>$ $c_{\exp }>\lambda(G) / 4$, and with at least $k / c$ and at most $k / 6$ vertices. We set $\alpha_{\mathrm{GT}}$ to be equal to $\min \left(\log c / c, \alpha_{\mathrm{CSP}} \cdot\left(1-c_{\mathrm{exp}}\right) / 16 c\right)$, where $\alpha_{\mathrm{CSP}}$ is the constant of Theorem 7. If $k$ is smaller than $c$, such an expander may not exist in $\mathcal{H}$, but since $\alpha_{\mathrm{GT}} \cdot c / \log c<1$, the trivial linear lower bound for the 4-Regular Graph Tiling problem, which holds for any $k \geq 2$, is enough to conclude. If $k$ is at least $c$, observing that the polynomial-time reduction blows up the number of vertices by 6 , we have that an algorithm deciding all the 4-REGULAR Graph TILING bipartite instances with at most $k$ vertices in time $O\left(n^{\alpha_{\mathrm{GT}} \cdot k / \log k}\right)$ would decide binary CSP instances whose primal graph is $G$ in time

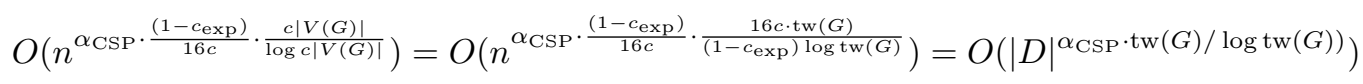

where the first equality uses Lemma 5 . This would contradict Theorem 7. 
- Remark 10. It might seem more natural to use a definition of 4-REgular GraPh Tiling where half-edges are labeled by $U, D, L$ and $R$, so that every edge contains either $U$ and $D$, or $L$ and $R$ labels. This fits more the intuition that the top side of a vertex should be attached to the bottom side of the next vertex. It follows from roughly the same proof that the same hardness result also holds for that variant. However, it seems that both the bipartiteness and the unusual labeling are required for the reduction in Section 4.

\section{$4 \quad$ Multiway cut with four terminals}

In this section, we prove the following proposition, which will yield Theorem 2 in the regime where the genus dominates the number of terminals.

\section{- Proposition 11.}

1. The Multiway Cut problem when restricted to instances $(G, T, \lambda)$ in which $|T|=4$ and $G$ is embeddable on the surface of genus $g$ is W[1]-hard parameterized by $g$.

2. Assuming ETH, there exists a universal constant $\alpha_{\mathrm{MC1}}$ such that for any fixed integer $g \geq 2$, there is no algorithm that decides all the Multiway Cut instances $(G, T, \lambda)$ for which $G$ is embeddable on the surface of genus $g$ and $|T|=4$, in time $O\left(n^{\alpha_{\mathrm{MC} 1} \cdot g / \log g}\right)$.

Proof. The idea is to reduce 4-Regular Graph Tiling instances of Theorem 9 to the instances of MultiwaY CUT specified by the proposition. Consider an instance of 4Regular Graph Tiling where the underlying graph $\Gamma$ is bipartite and has at most $k$ vertices ( $k$ being arbitrary for now). In polynomial time, we transform it into an equivalent instance $(G, T, \lambda)$ of Multiway Cut as follows.

1. To each vertex $v$ of $\Gamma$ corresponds a cross gadget $G_{S}(v)$ where $\Delta=n$ and the subset $S$ is chosen to be $S_{v}$.

2. For each edge $e=u v$ of $\Gamma$ labeled $U$, we identify the vertices of the $U$ side of the cross gadget $G_{S}(v)$ to the corresponding vertices of the $U$ side of the cross gadget $G_{S}(u)$. Similarly for the edges labeled $D, R$, and $L$ for which the vertices on the $D, R$, and $L$ sides, respectively, are identified. Note that only vertices, and not edges, are identified.

3. The four corner vertices $U L, U R, D R$, and $D L$ of all the cross gadgets are identified in four vertices $U L, U R, D R$, and $D L$, where the four terminals are placed.

Note that since the sides are consistently matched in this last step, the four terminals remain distinct after this identification.

We claim that this instance admits a multiway cut of weight at most $D_{2}:=k D_{1}$ (where $D_{1}$ is the integer from Lemma 8) if and only if the 4-Regular GraPh Tiling instance is satisfiable. Assume first that the 4-REgular GraPh Tiling instance is satisfiable. For each vertex $v$ of $\Gamma$, one can use the value $s_{v}$ to choose, using Lemma 8(1), a multiway cut in $G_{S}(v)$ representing $s_{v}$. We claim that the construction ensures that taking the union of all these sets of edges forms a multiway cut $M$ separating the four terminals in $G$. Indeed, after removing the multiway cuts, the four terminals lie in four different components in each of the cross gadgets. This remains the case after identifying the four sides: consider two cross gadgets that have two sides identified; let $w$ be a vertex on that common side; then, by the compatibility conditions in the definition of 4-REgular Graph Tiling, $w$ is connected, in the first gadget, to a terminal $(U L, U R, D R$, or $D L)$ if and only if it is connected to the corresponding terminal in the second gadget. The multiway cut $M$ has weight at most $k D_{1}$, since it is the union of $k$ edge sets of weight at most $D_{1}$.

For the other direction, we first observe that if the instance admits a multiway cut of weight at most $k D_{1}$, then each of the cross gadgets $G_{S}$ must admit a multiway cut (otherwise the four terminals would not be disconnected). By Lemma 8(2), each of these $k$ multiway cuts 


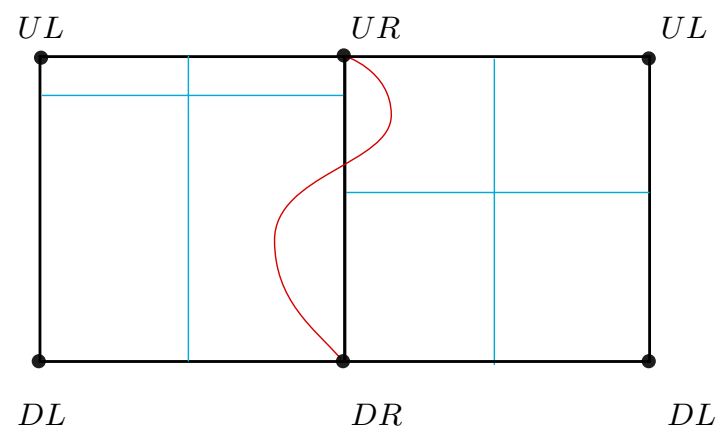

Figure 3 If the multiway cuts do not match (here represented by their duals), they do not separate the terminals.
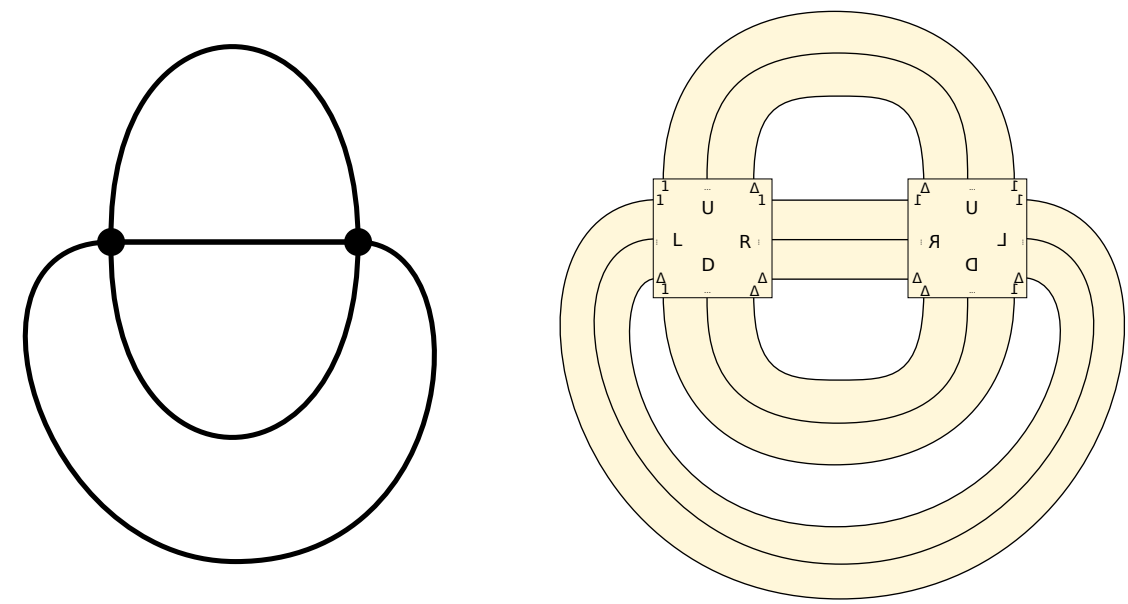

Figure 4 Left: A bipartite four-valent graph $\Gamma$ with two vertices. Right: The construction of the embedding of $G$. The orientation of each gadget of $G$, corresponding to a vertex $v$, is chosen according to the side of the bipartition vertex $v$ lies in. This allows to connect pairs of vertices on the boundary of each gadget with the same indices.

has weight exactly $D_{1}$. Therefore, by Lemma $8(3)$, each of them represents some $(i, j) \in S$, which will be used as the value $s_{v}$ for the 4-Regular Graph Tiling instance. Furthermore, we claim that the multiway cuts need to match along identified sides, i.e., if a multiway cut represents the pair $(i, j)$, then a multiway cut in a cross gadget adjacent along an edge labeled $U$ or $D$ needs to represent a pair $(k, j)$ for some $k \in[n]$, and similarly a multiway cut in a cross gadget adjacent along an edge labeled $R$ or $L$ needs to represent a pair $(i, \ell)$ for some $\ell \in[n]$, for otherwise the four terminals are not separated. Indeed, if, say, a multiway cut representing the pair $(i, j)$ is connected along an edge labeled $R$ to a multiway cut representing the pair $\left(i^{\prime}, \ell\right)$ for $i^{\prime}>i$, there is a path connecting the terminals $U R$ and $D R$, as pictured in Figure 3, contradicting the fact that we have a multiway cut. Therefore, the compatibility conditions of the 4-REGULAR GRAPH TILING instance are satisfied.

$\triangleright$ Claim 12. The genus of the graph $G$ is $O(k)$.

This claim is proved by providing an embedding of $G$, by connecting cross gadgets with at most $k$ ribbons which will then be contracted. An important subtlety is that the naive way of doing so does not yield an orientable surface, and to fix this, the fact that $G$ is bipartite turns out to be crucial: Our embedding switches the orientation of the gadgets based on the bipartition of the vertices (as pictured in Figure 4). The full proof of this claim is included in the full version $[4$, Section 4]. 

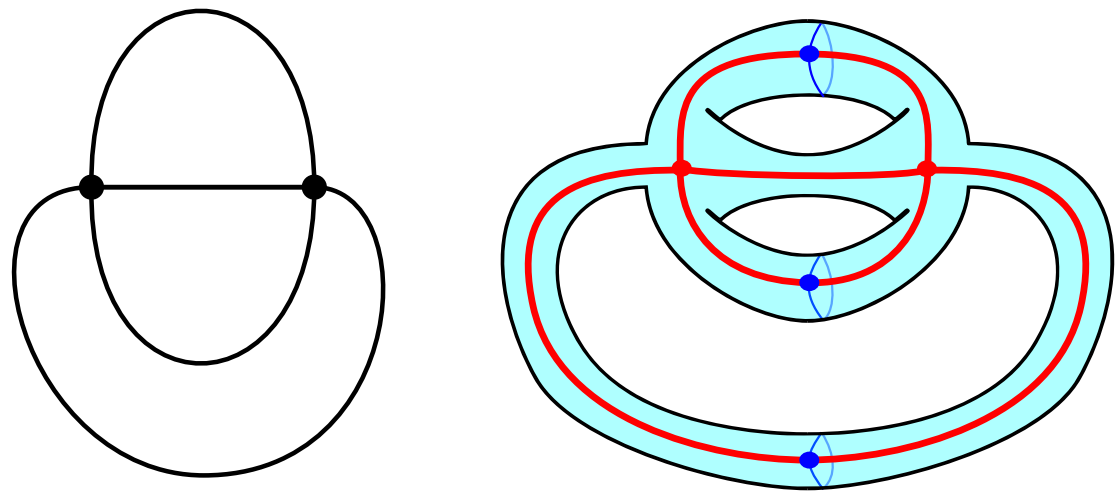

Figure 5 Left: A bipartite four-valent graph $\Gamma$ with two vertices. Right: The resulting graph $\Gamma^{\prime}$. The graph $\Gamma$ is in thick lines, and the edges forming the complement of a spanning tree are split with a new vertex, to which a loop (in thin lines) is attached.

To summarize: Given an instance of 4-REgular GraPh TiLING where the underlying graph $\Gamma$ is bipartite and has at most $k$ vertices, for an arbitrary $k$, we can transform it in polynomial time into an equivalent instance of MULTiwaY CUT with four terminals and whose graph has at most $k \cdot \operatorname{poly}(n)$ vertices and edges and is embeddable on a surface of genus at most $c k$, for some universal constant $c \geq 1$, where the polynomial is inherited from Lemma 8.

Combined with Theorem 9(1), this proves the first item. For the second one, for a given choice of $g$, we pick $k=\lfloor g / c\rfloor$. If $k \geq 2$, setting $\alpha_{\mathrm{MC} 1}=\alpha_{\mathrm{GT}} / c d$ where $d$ is the degree of the polynomial and combining Theorem 9(2) with this reduction proves the second item of the theorem. Otherwise, as in the proof of Theorem 9, choosing $\alpha_{\mathrm{MC} 1}$ to be smaller than $2 c / \log (2 c)$ and using the trivial linear lower bound suffices to conclude.

\section{Shortest cut graph}

In this section, we prove Theorem 1 on the hardness of the Shortest Cut GraPh problem.

Proof. The idea is to reduce 4-Regular Graph Tiling instances of Theorem 9 to instances of Shortest Cut Graph. Let $\Gamma$ be a bipartite four-regular graph with $k$ vertices.

From $\Gamma$, we build a surface $S$ as follows (see Figure 5): We build one cylindrical tube for each edge of $\Gamma$ and one sphere minus four disks for each vertex of $\Gamma$, attaching them in the natural way to obtain an orientable surface. By Euler's formula, this surface has genus $k+1$. Moreover, the graph $\Gamma$ is naturally embedded in $S$, though not cellularly. In order to have a cellular embedding, and actually a cut graph, we transform $\Gamma$ as follows. Let $T$ be a spanning tree of $\Gamma$. Let $\Gamma^{\prime}$ be the graph obtained from $\Gamma$ by subdividing each edge not in $T$ into two edges, and adding a loop in the middle vertex. Now, embed $\Gamma^{\prime}$ into $S$ in the natural way: Starting from the embedding of $\Gamma$ into $S$, put each middle vertex on the corresponding cylindrical tube of $S$, and make the corresponding loop go around the tube. The resulting graph is a cut graph of $S$ (indeed, it has a single face, because we only add loops in the middle of edges not in the spanning tree $T$; $\Gamma^{\prime}$ has $2 k+1$ vertices and $4 k+2$ edges (being four-regular); so its unique face is a disk, by Euler's formula).

Let $V_{1} \cup V_{2}$ be the bipartition of the vertices of $\Gamma$. We note that the above construction is possible while enforcing an arbitrary cyclic ordering of the edges incident to each vertex of $\Gamma$; we do it in a way that the cyclic ordering of the edges around each vertex in $V_{1}$ is the standard one ( $U, R, D, L$ in clockwise order), while the cyclic ordering around each vertex 
in $V_{2}$ is reversed ( $U, L, D, R$ in clockwise order). We now build a graph $G$ embedded on the same surface $S$ as follows, obtained by replacing each vertex of $\Gamma^{\prime}$ with a dual cross gadget and by (almost) identifying vertices on the corresponding sides of adjacent gadgets. In detail: 1. To each vertex $v$ of $\Gamma$ corresponds a dual cross gadget $G_{S}^{*}(v)$ where $\Delta=n$ and the subset $S$ is chosen to be $S_{v}$. We embed that dual cross gadget with the same orientation as the corresponding vertex of $\Gamma^{\prime}$.

2. For each edge $e=u v$ of $T$, we identify the vertices (not the edges) on the side of $G_{S}^{*}(u)$ corresponding to the label of $e$ to the vertices on the same side of $G_{S}^{*}(v)$. By the choice of the rotation systems, and for the same reason as in Figure 4, this identifies the vertices in the gadget associated with $u$ to the corresponding vertices in the gadget associated with $v$; for example, if the label of edge $e$ is $R$, the vertex $r_{i}$ of the first gadget is associated to vertex $r_{i}$ of the second gadget).

3. For an edge $e=u v$ of $\Gamma$ not in $T$, we use another dual cross gadget $G_{S}^{*}(e)$, for which we choose $S$ to be the unconstrained relation $S=[n]^{2}$. We put that gadget on the vertex of $\Gamma^{\prime}$. We identify the vertices on the side of $G_{S}^{*}(u)$ corresponding to the label of $e$ to the vertices of the same side of $G_{S}^{*}(e)$, and similarly the vertices on the side of $G_{S}^{*}(v)$ corresponding to the label of $e$ to the vertices on the opposite side of $G_{S}^{*}(e)$. The two opposite sides of $G_{S}^{*}(e)$ which are not yet identified are identified to each other.

The following claim, whose proof is included in the full version [4, Section 5], shows that the reduction works as expected.

$\triangleright$ Claim 13. The embedded graph $G$ admits a cut graph $C$ of weight at most $(2 k+1) D_{1}$ if and only if the 4-Regular Graph Tiling instance on $\Gamma$ is satisfiable.

To summarize: Given an instance of 4-REgular Graph TILING where the underlying graph $\Gamma$ is bipartite and has $k$ vertices, for an arbitrary $k$, we can transform it in polynomial time into an equivalent instance of SHORTEST CUT Graph whose graph has $k \cdot \operatorname{poly}(n)$ vertices and edges, embedded on a surface of genus $k+1$. Combined with Theorem 9(1), this proves the first item of the theorem. For the second item, for any choice of integer $g \geq 3$, we choose $k=g-1$, and the above reduction, combined with Theorem 9(2), finishes the proof for $\alpha_{\mathrm{CG}} \leq \alpha_{\mathrm{GT}} / d$ (where $d$ is the degree of the polynomial of Lemma 8). For $g=2$, we set $\alpha_{\mathrm{CG}} \leq 2$ and conclude using the trivial linear lower bound.

\section{Multiway cut with a large number of terminals}

The goal of this section is to prove the following proposition, which yields Theorem 2 when the number of terminals dominates the genus. Recall that $g^{*}$ denotes $\max (g, 2)$.

- Proposition 14. Assuming ETH, there exists a universal constant $\alpha_{\mathrm{MC} 2}$ such that for any fixed choice of integers $g \geq 0$ and $t \geq 24 g^{*}$, there is no algorithm that decides all the Multiway Cut instances $(G, T, \lambda)$ for which $G$ is embeddable on the surface of genus $g$ and $|T| \leq t$, in time $O\left(n^{\alpha_{\mathrm{MC} 2} \sqrt{g^{*} t} / \log \left(g^{*} t\right)}\right)$.

Sketch of proof. We only sketch the proof and refer to the full version [4, Section 6] for details. Here are the key ideas: The reduction bypasses the use of 4-REgular GrID TILING and instead starts directly from a binary CSP instance on a four-regular graph $P$. In a way similar to the proofs of Proposition 11 and Theorem 1, this instance can be encoded in a Multiway Cut instance by using cross gadgets to encode the constraints. In order to obtain the claimed lower bound, we will apply Theorem 7, and thus we need to choose for 

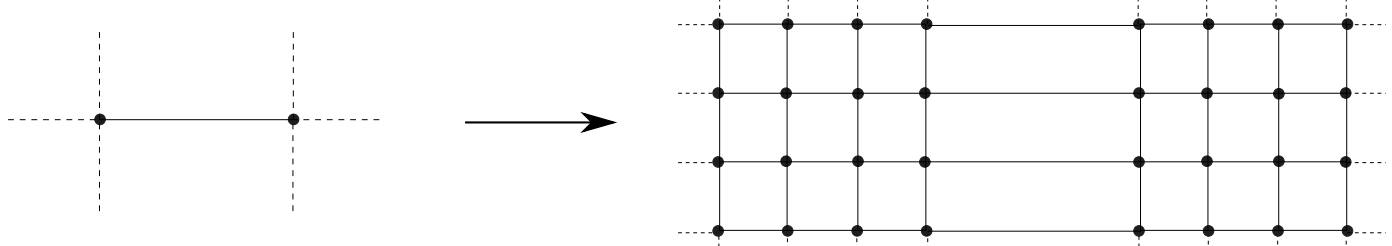

Figure 6 The construction of $G_{\delta}$ for $\delta=4$.

$P$ a graph having genus at most $g^{*}$ and treewidth $\Omega\left(g^{*} t\right)$; our construction uses a blow-up of an expander graph, i.e., an expander graph where each vertex has been replaced with a grid of an appropriate size (see Figure 6) - this is a construction reminiscent of one used in Gilbert, Hutchinson and Tarjan [15].

Finally, the proof of Theorem 2 is obtained by using Proposition 11 or Proposition 14, depending on the tradeoff between $g$ and $t$. This is carried out in the full version [4, Section 7].

\section{References}

1 Jianer Chen, Iyad A Kanj, Ljubomir Perković, Eric Sedgwick, and Ge Xia. Genus characterizes the complexity of certain graph problems: Some tight results. Journal of Computer and System Sciences, 73(6):892-907, 2007.

2 Rajesh Hemant Chitnis, MohammadTaghi Hajiaghayi, and Dániel Marx. Tight Bounds for Planar Strongly Connected Steiner Subgraph with Fixed Number of Terminals (and Extensions). In 25th ACM-SIAM Symposium on Discrete Algorithms (SODA), pages 1782-1801, 2014. doi:10.1137/1.9781611973402.129.

3 Vincent Cohen-Addad, Éric Colin de Verdière, and Arnaud de Mesmay. A near-linear approximation scheme for multicuts of embedded graphs with a fixed number of terminals. In 29th ACM-SIAM Symposium on Discrete Algorithms (SODA), pages 1439-1458, 2018.

4 Vincent Cohen-Addad, Éric Colin de Verdière, Dániel Marx, and Arnaud de Mesmay. Almost Tight Lower Bounds for Hard Cutting Problems in Embedded Graphs. Full version of this article, 2019. arXiv: 1903.08603.

5 Vincent Cohen-Addad and Arnaud de Mesmay. A fixed parameter tractable approximation scheme for the optimal cut graph of a surface. In European Symposium on Algorithms (ESA), pages 386-398. Springer, 2015.

6 Éric Colin de Verdière. Multicuts in planar and bounded-genus graphs with bounded number of terminals. Algorithmica, 78(4):1206-1224, 2017.

7 Éric Colin de Verdière. Computational topology of graphs on surfaces. In Jacob E. Goodman, Joseph O'Rourke, and Csaba Toth, editors, Handbook of Discrete and Computational Geometry, chapter 23, pages 605-636. CRC Press LLC, third edition, 2018.

8 Radu Curticapean and Dániel Marx. Tight conditional lower bounds for counting perfect matchings on graphs of bounded treewidth, cliquewidth, and genus. In 27th ACM-SIAM symposium on Discrete algorithms (SODA), pages 1650-1669. Society for Industrial and Applied Mathematics, 2016.

9 Marek Cygan, Fedor V Fomin, Łukasz Kowalik, Daniel Lokshtanov, Dániel Marx, Marcin Pilipczuk, Michał Pilipczuk, and Saket Saurabh. Parameterized algorithms, volume 3. Springer, 2015.

10 Elias Dahlhaus, David S. Johnson, Christos H. Papadimitriou, Paul D. Seymour, and Mihalis Yannakakis. The complexity of multiterminal cuts. SIAM Journal on Computing, 23(4):864894, 1994 
11 Mark de Berg, Hans L. Bodlaender, Sándor Kisfaludi-Bak, Dániel Marx, and Tom C. van der Zanden. A framework for ETH-tight algorithms and lower bounds in geometric intersection graphs. In 50th ACM Symposium on Theory of Computing (STOC), pages 574-586, 2018.

12 Erik D Demaine, Fedor V Fomin, Mohammadtaghi Hajiaghayi, and Dimitrios M Thilikos. Subexponential parameterized algorithms on bounded-genus graphs and H-minor-free graphs. Journal of the ACM, 52(6):866-893, 2005.

13 Jeff Erickson and Sariel Har-Peled. Optimally cutting a surface into a disk. Discrete \& Computational Geometry, 31(1):37-59, 2004.

14 Fedor V. Fomin, Daniel Lokshtanov, Dániel Marx, Marcin Pilipczuk, Michal Pilipczuk, and Saket Saurabh. Subexponential Parameterized Algorithms for Planar and Apex-Minor-Free Graphs via Low Treewidth Pattern Covering. In IEEE 57th Symposium on Foundations of Computer Science, FOCS, pages 515-524, 2016. doi:10.1109/FOCS.2016.62.

15 John R. Gilbert, Joan P. Hutchinson, and Robert Endre Tarjan. A separator theorem for graphs of bounded genus. Journal of Algorithms, 5(3):391-407, 1984.

16 Martin Grohe. The complexity of homomorphism and constraint satisfaction problems seen from the other side. Journal of the ACM, 54(1):1, 2007.

17 Martin Grohe, Thomas Schwentick, and Luc Segoufin. When is the evaluation of conjunctive queries tractable? In 33rd ACM Symposium on Theory of Computing (STOC), pages 657-666, 2001.

18 Te C. Hu. Integer programming and network flows. Technical report, Wisconsin Univ Madison Dept. of Computer Sciences, 1969.

19 Russell Impagliazzo, Ramamohan Paturi, and Francis Zane. Which problems have strongly exponential complexity? In 39th IEEE Symposium on Foundations of Computer Science (FOCS), pages 653-662, 1998.

20 Ken-ichi Kawarabayashi, Bojan Mohar, and Bruce Reed. A simpler linear time algorithm for embedding graphs into an arbitrary surface and the genus of graphs of bounded tree-width. In 49th IEEE Symposium on the Foundations of Computer Science (FOCS), pages 771-780. IEEE, 2008.

21 Philip N. Klein and Dániel Marx. Solving Planar $k$-Terminal Cut in $O\left(n^{c \sqrt{k}}\right)$ Time. In International Colloquium on Automata, Languages, and Programming (ICALP), pages 569-580. Springer, 2012.

22 Daniel Lokshtanov, Dániel Marx, Saket Saurabh, et al. Lower bounds based on the exponential time hypothesis. Bulletin of EATCS, 3(105), 2013.

23 Daniel Lokshtanov, Saket Saurabh, and Magnus Wahlström. Subexponential Parameterized Odd Cycle Transversal on Planar Graphs. In IARCS Annual Conference on Foundations of Software Technology and Theoretical Computer Science, FSTTCS 2012, December 15-17, 2012, Hyderabad, India, pages 424-434, 2012. doi:10.4230/LIPIcs.FSTTCS. 2012.424.

24 Dániel Marx. On the Optimality of Planar and Geometric Approximation Schemes. In 48th IEEE Symposium on Foundations of Computer Science (FOCS'07), pages 338-348, 2007.

25 Dániel Marx. Can You Beat Treewidth? Theory of Computing, 6(1):85-112, 2010.

26 Dániel Marx. A Tight Lower Bound for Planar Multiway Cut with Fixed Number of Terminals. In Artur Czumaj, Kurt Mehlhorn, Andrew Pitts, and Roger Wattenhofer, editors, Automata, Languages, and Programming, pages 677-688, Berlin, Heidelberg, 2012. Springer Berlin Heidelberg.

27 Dániel Marx. The Square Root Phenomenon in Planar Graphs. In Frontiers in Algorithmics and Algorithmic Aspects in Information and Management, volume 7924. Springer, 2013.

28 Dániel Marx, Marcin Pilipczuk, and Michał Pilipczuk. On subexponential parameterized algorithms for Steiner Tree and Directed Subset TSP on planar graphs. In 59th IEEE Symposium on Foundations of Computer Science (FOCS), pages 474-484, 2018.

29 Dániel Marx and Michał Pilipczuk. Everything you always wanted to know about the parameterized complexity of Subgraph Isomorphism (but were afraid to ask). In 31st International Symposium on Theoretical Aspects of Computer Science (STACS), volume 25, pages 542-553, 2014 . 
30 Dániel Marx and Michal Pilipczuk. Optimal Parameterized Algorithms for Planar Facility Location Problems Using Voronoi Diagrams. In 23rd European Symposium on Algorithms (ESA), pages 865-877, 2015.

31 Dániel Marx and Anastasios Sidiropoulos. The limited blessing of low dimensionality: when $1-1 / d$ is the best possible exponent for $d$-dimensional geometric problems. In 30th Symposium on Computational Geometry (SoCG), page 67. ACM, 2014.

32 Bojan Mohar. A linear time algorithm for embedding graphs in an arbitrary surface. SIAM Journal on Discrete Mathematics, 12(1):6-26, 1999.

33 Bojan Mohar and Carsten Thomassen. Graphs on surfaces. Johns Hopkins Studies in the Mathematical Sciences. Johns Hopkins University Press, 2001.

34 Zoë Wood, Hugues Hoppe, Mathieu Desbrun, and Peter Schröder. Removing excess topology from isosurfaces. ACM Transactions on Graphics (TOG), 23(2):190-208, 2004. 